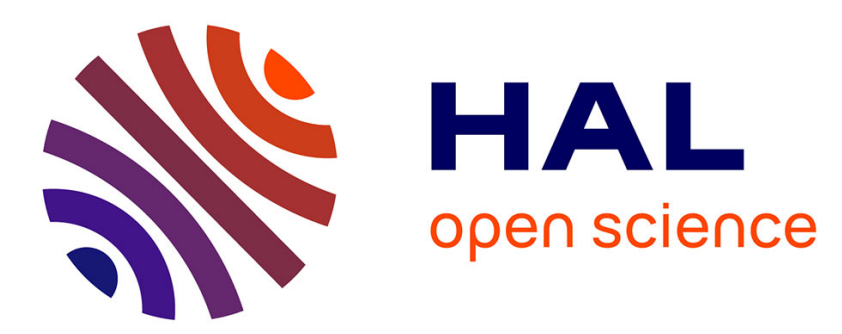

\title{
Numerical studies of dielectric material modifications by a femtosecond Bessel-Gauss laser beam
}

\author{
R. Beuton, B. Chimier, P. Quinoman, P. González Alaiza de Martínez, R. \\ Nuter, G. Duchateau, P González de Alaiza Martínez
}

\section{- To cite this version:}

R. Beuton, B. Chimier, P. Quinoman, P. González Alaiza de Martínez, R. Nuter, et al.. Numerical studies of dielectric material modifications by a femtosecond Bessel-Gauss laser beam. Applied physics. A, Materials science \& processing, 2021, 127 (5), 10.1007/s00339-021-04476-y . hal03394790

\author{
HAL Id: hal-03394790 \\ https://hal.science/hal-03394790
}

Submitted on 25 Oct 2021

HAL is a multi-disciplinary open access archive for the deposit and dissemination of scientific research documents, whether they are published or not. The documents may come from teaching and research institutions in France or abroad, or from public or private research centers.
L'archive ouverte pluridisciplinaire HAL, est destinée au dépôt et à la diffusion de documents scientifiques de niveau recherche, publiés ou non, émanant des établissements d'enseignement et de recherche français ou étrangers, des laboratoires publics ou privés. 


\title{
Numerical studies of dielectric material modifications by a femtosecond Bessel-Gauss laser beam
}

\author{
R. Beuton - B. Chimier - P. Quinoman - P. González de Alaiza \\ Martínez • R. Nuter • G. Duchateau
}

Received: date / Accepted: date

\begin{abstract}
Femtosecond Bessel-Gauss beams are attractive tools to a large area of laser processes including high aspect ratio volume nanostructuration in dielectric materials. Understanding the dielectric material response to femtosecond Bessel-Gauss beam irradiation is key in controlling its modifications and designing new structures. In this work, we show how the material ionization affects the propagation of the femtosecond Bessel-Gauss laser beam and can limit the laser energy deposition. By performing 2D/3D numerical simulations, we evaluate the absorbed laser energy and subsequent material modifications. First, we model the electron dynamics in the material coupled to the $3 \mathrm{D}$ laser propagation effects. Then, we consider $2 \mathrm{D}$ thermoelasto-plastic simulations to characterize the medium modifications. Results show that the laser ionized matter induces a screening of the incident gaussian beams which form the Bessel-Gauss beam. This effect leads to a limitation of the maximum laser energy deposition even if the incident laser energy increases. It can be reduced if a tigthly focused femtosecond Bessel-Gauss beam is used as the angular aperture of the cone along which the incident gaussian beams are distributed is larger.
\end{abstract}

\section{Introduction}

Femtosecond laser pulse is a versatile tool for processing of dielectric materials [1-4] due to a highly nonlinear laser energy absorption and an irradiation time

R. Beuton · B. Chimier · P. Quinoman · P. González de Alaiza Martínez · R. Nuter · G. Duchateau

Université de Bordeaux - CNRS - CEA, Centre Lasers Intenses et Applications, UMR 5107, 33405 Talence, France shorter than typical hydrodynamic and thermal relaxation times in dielectrics $(\sim 10 \mathrm{ps})$. This fast heating process provides an efficient way to induce material modifications in the bulk of dielectrics, and has numerous applications in many fields, such as photonics or micromachining [5]. But, the size of laser induced structures is limited by the focal volume which is small for traditionnal Gaussian pulses as they must be tightly focused inside the dielectric $[6,7]$. This limitation can be overcome by using non-diffractive Bessel-Gauss beams. These beams correspond to long and narrow filaments along the optical axis with only hundreds of nanometers in diameter, and a length which may exceed several hundreds of microns [8-10].

A Bessel-Gauss beam is usually produced by using axicon and results from interferences of incident Gaussian beam with itself $[11,12]$. The laser pulse energy propagates along directions distributed on a cone surface with a given angle $\theta$ with the axial propagation direction, and converge to a line. For dielectric material modifications, the formed Bessel-Gauss beam is then projected inside the matter by a $4 f$ afocal imaging system $[13,14]$ and on-axis intensity may be tailored by using spacial light modulators or filters $[15,16]$. For this kind of nonpropagative beam, as the laser energy does not propagate on propagation axis, it is generally assumed that the laser energy can be absorbed in the matter along the whole length of the imaged beam. Depending on the incident laser energy, modifications in the whole irradiated volume, like refractive index variation or void structure formation, can be obtained [1-3,17], which is of interest for high aspect ratio, uniform, and submicron structuring of transparent materials by using a single laser pulse. However, for femtosecond Bessel-Gauss laser pulses, void structures in bulk of fused silica can be achieved only in tight fo- 
cusing conditions $[3,18]$. A large $\theta$ value leading to a Bessel-Gauss beam core of submicrometer full width at half-maximum is necessary. An influence of the optical material response (optical index change inducing diffraction, plasma screening, etc) has been suggested to affect the laser pulse propagation and limit the laser energy deposition. This effect is assumed to decrease since the $\theta$ value increases. Understanding this limitation of laser energy absorption is therefore necessary to control and improve material processing by using femtosecond Bessel-Gauss beams.

To understand and explain such experimental observations in femtosecond regime, material modifications in fused silica by $60 \mathrm{fs}$ and $800 \mathrm{~nm}$ Bessel-Gauss laser pulses are numerically studied in the present work. These laser parameters correspond to those used in $[\mathbf{3}, \mathbf{1 8}]$. Three different $\theta$ values are considered to evaluate the influence of the Bessel-Gauss beam diameter. Due to the different timescales involved during the material modification, electron dynamics including laser energy absorption and electron energy transfer toward the lattice, can be decoupled from hydrodynamic processes and thermal conduction [7]. The electron dynamics coupled to the propagation of BesselGauss beam is widely modeled by solving the nonlinear Schrödinger equation supplemented with a rate equation describing ionization and recombination of conduction band electrons $[2,14,19]$. However, the nonlinear Schrödinger equation is based on approximations of slowly varying envelope of laser pulse and light scattering limited to small angles. These assumptions are valid as long as the conduction electron density in the irradiated matter remains lower than the critical density during the laser pulse propagation, that means to moderate laser intensities for which only small modifications are expected. In this work, to address the formation of cavity or channel inside dielectric material which require high intensities and possibly electron densities in excess of the critical density, full Maxwell's equations coupled to the electron dynamics are used to model the laser pulse propagation and interaction. A two temperature fluid model including ionization processes has been introduced in a 3D Maxwell solver (ARCTIC code [20]) and is presented in Sec. 2. The evolution of the electron dynamics and laser energy deposition in the bulk are studied in Sec. 3 for femtosecond Bessel-Gauss beams with different beam diameters. Influence of the material ionization on the Bessel-Gauss laser beam formation is shown and discussed. A limitation of the maximum material laser heating depending of the beam diameter is observed. The calculated absorbed energy profile is then used as initial condition in the hydrodynamic code CHIC [21] including the elasto-plastic behaviour of the solid matter [22-24]. It provides material modifications post to the interaction, which are presented in Sec. 4 . In agreement with experimental results $[3,18]$, the smaller the Bessel-Gauss beam diameter (tighter focusing condition), the stronger the material modification.

\section{Modeling of laser propagation and energy deposition}

The Maxwell's equations for the laser pulse propagation through a dielectric medium read:

$\boldsymbol{\nabla} \wedge \mathbf{E}=-\partial_{t} \mathbf{B}$ and $\mu_{0}^{-1} \nabla \wedge \mathbf{B}=\partial_{t} \mathbf{D}+\mathbf{J}$,

where $\mathbf{E}, \mathbf{D}, \mathbf{B}$, and $\mu_{0}$ are the laser electric field, the electric displacement field, the magnetic field, and the vacuum permeability, respectively. The current $\mathbf{J}=$ $\mathbf{J}_{I}+\mathbf{J}_{e}$, where $\mathbf{J}_{I}$ is the effective ionization current depending on the material bandgap and photoionization rate [25] and $\mathbf{J}_{e}=-e n_{e} \mathbf{u}_{e}$ is the free electron current density $\left(n_{e}\right.$ and $\mathbf{u}_{e}$ are the conduction electron density and velocity, respectively). The electric displacement field D accounts for material polarization through linear and nonlinear dielectric susceptibilities [25] $\chi^{(1)}$ and $\chi^{(3)}$, respectively.

The electron dynamics in the conduction band and lattice heating are described by using a two-temperature fluid model assuming isochoric processes and neglecting thermal conduction. The electron momentum equation is written as

$\partial_{t} \mathbf{u}_{e}=-e \mathbf{E} / m_{e}-\mathbf{u}_{e} \nu_{e}$,

where $m_{e}$ is the electron mass, and $\nu_{e}$ is the effective electron collision frequency. The evolution of the electron density in the conduction band is given by

$\partial_{t} n_{e}=W_{P I}+W_{C o l}-n_{e} / \tau_{r}$,

where the last term accounts for the electron decay with the characteristic time $\tau_{r}$, the photoionization rate $W_{P I}$ is given by the Keldysh expression for solids [26], and the impact ionization rate $W_{C o l}$ is evaluated by $[27,28]$

$W_{C o l}=\alpha_{0} \int\left(\frac{\varepsilon}{U_{g}}-1\right)^{2} f_{e}(\varepsilon) d \varepsilon$,

where $\alpha_{0}$ is a constant rate depending on material, $U_{g}$ is the material bandgap, and $f_{e}$ is the electron energy distribution which is assumed to be a Maxwellian in this study.

The internal electron energy $\xi_{e}$ is given by $\partial_{t}\left(n_{e} \xi_{e}\right)=n_{e} u_{e}^{2} \nu_{e}-(3 / 2) U_{g} W_{C o l}-\gamma_{e l}\left(T_{e}-T_{l}\right)$,

where the two last terms represent the energy lost due to collisional ionization and to the lattice heating process, respectively. $\gamma_{e l}=3 n_{e} k_{B} /\left(2 \tau_{e l}\right)$ is the electronlattice coupling where $\tau_{e l}$ is the electron-lattice relaxation time, and $T_{e}$ and $T_{l}$ are the electron and lattice 
Table 1: Diameters $d_{0}$, widths of the gaussian term $w_{0}$, and angular half apertures $\theta$ of the three considered Bessel-Gauss beams with $20 \mu \mathrm{m}$ in length.

\begin{tabular}{cccc}
\hline Beam & $d_{0}(\mu \mathrm{m})$ & $w_{0}(\mu \mathrm{m})$ & $\theta\left(^{\circ}\right)$ \\
\hline BG1 & 3.11 & 2 & 11.3 \\
BG2 & 1.8 & 3.5 & 19.3 \\
BG3 & 1.3 & 5 & 26.5 \\
\hline
\end{tabular}

temperatures $\left(T_{e}=2 \xi_{e} /\left(3 n_{e} k_{B}\right)\right)$. The lattice temperature evolution is given by

$C_{l} \partial_{t} T_{l}=\gamma_{e l}\left(T_{e}-T_{l}\right)$,

where $C_{l}$ is the lattice heat capacity.

The previous equations are implemented in the code ARCTIC [20], where Maxwell equations (1) are discretized in the three spatial dimensions by means of Yee scheme [29]. The simulation domain includes Bérenger's Perfectly-Matched-Layer absorbing boundary condition [3 31]. The propagation axis is $x$.

Instead of determining the initial boundary conditions by simulating all the imaging system to produce a Bessel-Gauss beam, the analytical expression given in Ref [32] has been used. This expression corresponds to a Bessel-Gauss beam generated by using an annular aperture as filter $[15,16]$. In vacuum, the beam intensity is maximum in $x=0$, and

$\mathbf{E}(x=0, r, t)=\mathbf{E}_{0}(t) J_{0}(\beta r) \exp \left[-\left(r / w_{0}\right)^{2}\right]$,

where $J_{0}$ corresponds to the zero order Bessel function, $\mathbf{E}_{0}(t)$ includes the Gaussian temporal dependences, $r=$ $\sqrt{y^{2}+z^{2}}$ and $\beta=k \sin \theta, \theta$ being the angular half aperture of the cone along which the incident gaussian beams are distributed, and $k=2 \pi / \lambda_{l}$ ( $\lambda_{l}$ is the laser wavelength in vacuum). The Bessel-Gauss beam length is given by $D=2 w_{0} / \tan \theta$. From the electric field defined by Eq. (7), the initial boundary conditions at $x=-25 \mu \mathrm{m}$ in simulations are calculated by using the algorithm presented in Ref. $[33,34]$.

\section{Bessel-Gauss beam propagation in bulk of fused silica}

For simulation purpose, $60 \mathrm{fs}$ and $800 \mathrm{~nm}$ incident laser pulses are considered with maximum peak intensities $I_{0}$ at $x=0$ between 20 and $1000 \mathrm{TW} / \mathrm{cm}^{2}$ like in [3, 18]. Three Bessel-Gauss beams with different diameters $d_{0}$ (distance between the first zeros of the Bessel function in Eq. (7)) and a $20 \mu \mathrm{m}$ in length in vacuum are considered and presented in Tab. 1. The simulated Bessel-Gauss length is ten times smaller than the typical Bessel-Gauss length used in experiments [3,18] due to computational constraints. However, this length is enough to observe modifications of the matter which differ from traditional Gaussian pulses and to capture the main physical processes at play.

The Bessel-Gauss beams propagate inside fused silica target $[36,37]$ with initial solid density $\rho_{0}=2.2 \mathrm{~g} / \mathrm{cm}^{3}$, $U_{g}=9 \mathrm{eV}, \tau_{r}=150 \mathrm{fs}, \nu_{e}=5 \mathrm{fs}^{-1}, \tau_{e l}=1 \mathrm{ps}, C_{l}=$ $1.6 \mathrm{~J} / \mathrm{cm}^{3} / \mathrm{K}, \chi^{(1)}=1.11$ and $\chi^{(3)}=2 \times 10^{-22} \mathrm{~m}^{2} / \mathrm{V}^{2}$. Note that the optical index in the non ionized matter is different from the vacuum where both Bessel-Gauss beams have been initially defined. In the material, the position of maximum intensity in Bessel-Gauss beams is forward shifted by $11.3 \mu \mathrm{m}$ along the propagation axis.

The typical value of the $\alpha_{0}$ rate in the impact ionization model (see Eq. (4)) is of the order of $1.5 \mathrm{fs}^{-1}$ for fused silica $[27,28]$. However, using this value in our simulations with a $60 \mathrm{fs}$ and $800 \mathrm{~nm}$ laser pulse, leads to underestimate the absorbed laser energy, and thus the final lattice temperature. For the BG1 beam with $I_{0}=300 \mathrm{TW} / \mathrm{cm}^{2}$, the calculated final lattice temperature in the heated matter is in the range of 600 to $800 \mathrm{~K}$. The final lattice temperature should be in the range of the strain and annealing temperatures, that means of the order of $T_{s}=1300 \mathrm{~K}$ in fused silica [38], as structure inducing change of the refractive index is experimentally observed for such laser parameters [3]. This low heating in simulation is induced by the sharp evolution of the conduction band electron density leading to a strong screening of the incident laser beam as it will be demonstrated after. On the other hand, removing the impact ionization process in Eq. (4) results to calculated final lattice temperatures of the order of some $\mathrm{eV}$, which is to large. To obtain a temperature of the order of $T_{s}$ in the heated material, $\alpha_{0}$ must be of the order of $0.03 \mathrm{fs}^{-1}$ in our simulation. This decrease of the $\alpha_{0}$ value is in agreement with experiment and simulation results [39-41] showing a decrease of the importance of the impact ionization process for laser pulses shorter than 100 fs. This $\alpha_{0}$ value is used in all forthcoming simulations.

Figures 1 and 2 present the temporal and spatial evolutions of the electron density and laser intensity distributions in the $x y$ plane for the BG1 and BG3 beams, respectively. The maximum laser peak intensity in vacuum is $I_{0}=300 \mathrm{TW} / \mathrm{cm}^{2}$ in both cases. The laser pulse comes from the left border. The electron densities $\bar{n}_{e}$ are normalized to the critical electron density $n_{c}=1.7 \times 10^{21} \mathrm{~cm}^{-3}$. The black and white lines in Figs. 1(a) and 2(a), respectively, correspond to $n_{e}=0.5 n_{c}$. Larger densities provide an efficient laser energy absorption and reflectivity for the considered laser parameters. 

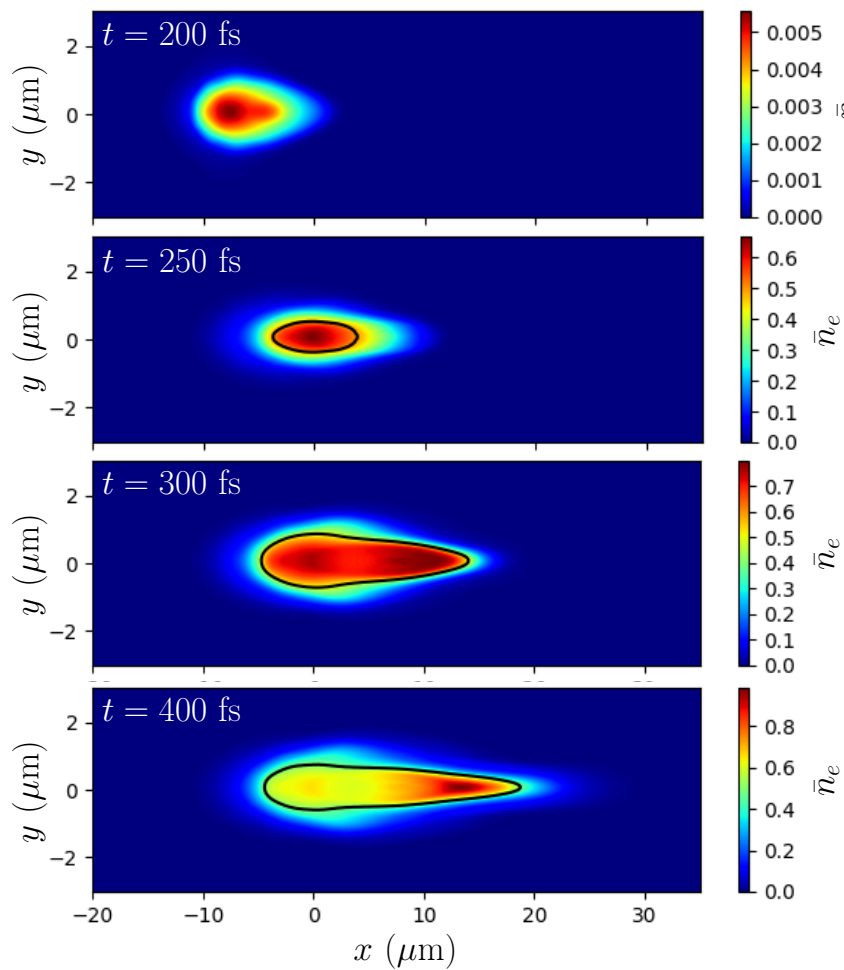

(a)
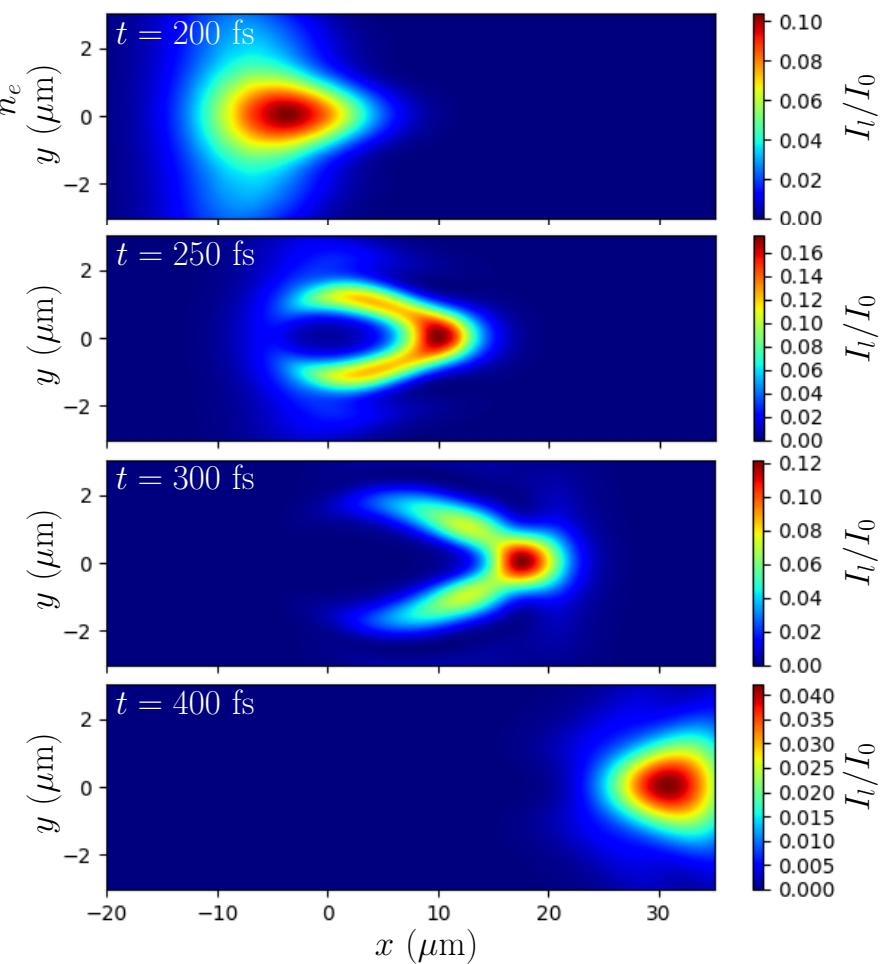

(b)

Fig. 1: Spatial and temporal evolutions in the xy plane of the free electron density (a) and laser intensity (b) in silica for $3.11 \mu \mathrm{m}$ in diameter Bessel-Gauss beam (BG1). The laser pulse comes from the left border and the laser intensity $I_{l}$ is normalized to the laser peak intensity $I_{0}=300 \mathrm{TW} / \mathrm{cm}^{2}$. The electron density $\bar{n}_{e}$ is normalized to $n_{c}=1.7 \times 10^{21} \mathrm{~cm}^{-3}$.

For both Bessel-Gauss beams, the electron density increases slowly during the first $200 \mathrm{fs}$ after the beginning of the laser irradiation, because only photoionization takes place. The electron density in the ionized matter is too low to significantly disturb the incident laser pulse propagation and the Bessel-Gauss beams begins to form in the interference area (around $y=0$ ) along the propagation axis. Thereafter, when the laser intensity becomes greater than $0.1 I_{0}$, the energy of the ionized electrons becomes large enough to induce collisional ionization. The electron density in the conduction band sharply increases and a volume of matter where $n_{e}>0.5 n_{c}$ appears.

For the BG1 beam (Fig. 1(a)), the transverse diameter of the absorption volume where $n_{e}>0.5 n_{c}$, is approximately $1 \mu \mathrm{m}$ and its length is $8 \mu \mathrm{m}$ for $t=250$ fs. Its shape evolves toward an elongated teardrop shape with $23.2 \mu \mathrm{m}$ in length and $1.3 \mu \mathrm{m}$ in maximum diameter at $t=400 \mathrm{fs}$. The maximum electron density is always lower than $n_{c}$. Appearance of the absorption volume disturbs the Bessel-Gauss beam formation (Fig. 1(b)). A large part of the incident beam is ab- sorbed and reflected by the ionized material before to reach the interference area. Only its unperturbed part, which propagates in matter where $n_{e}<0.5 n_{c}$, forms a small Bessel-Gauss beam in front of the absorption volume. The laser intensity is maximum in this area but always lower than $I_{0}$. This leads to a decrease of tranverse dimension of the absorption volume and to the elongated teardrop shape.

Concerning the BG3 beam (Fig. 2(a)), the maximum transverse size of the absorption volume where $n_{e}>0.5 n_{c}$ increases up to $1.4 \mu \mathrm{m}$, that means up to the size of the BG3 beam diameter, at $t=300 \mathrm{fs}$ and remains constant after while its length increases up to $30 \mu \mathrm{m}$ at $t=400 \mathrm{fs}$. At this time, the absorption volume is more homogeneous, thinner and longer than the absorption volume obtained with the BG1 beam. Like in the previous case, the laser beam propagation is also disturbed by the absorption volume formation (Fig. 2(b)). However, as the $\theta$ value is higher in the BG3 beam, the screening effect is lower. The maximum laser intensity in the small Bessel-Gauss beam in front of the absorption volume is always higher than in the BG1 

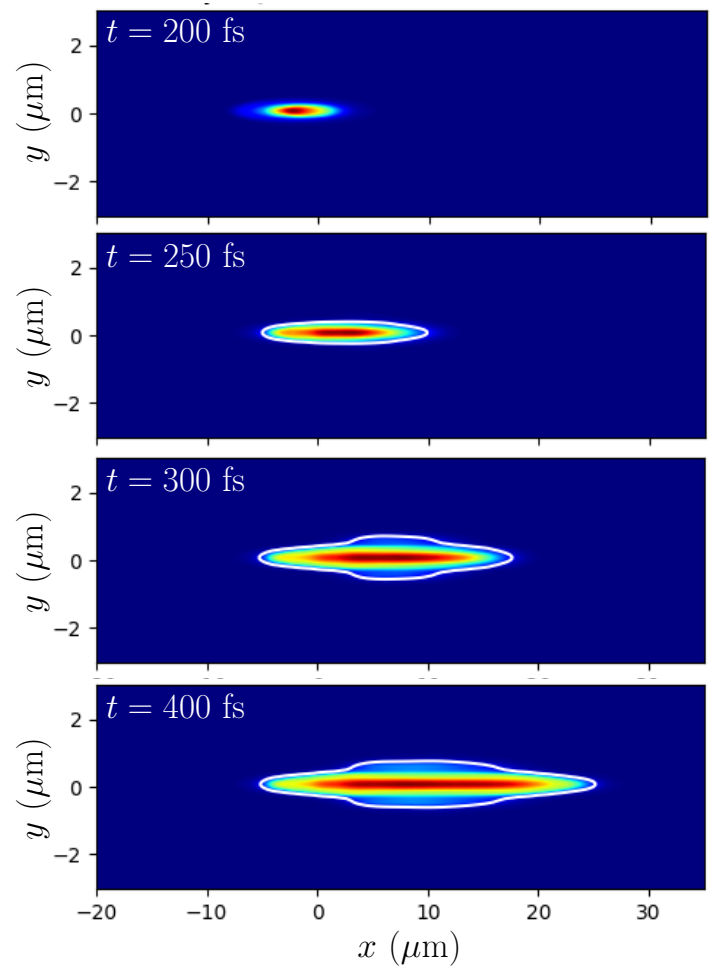

(a)
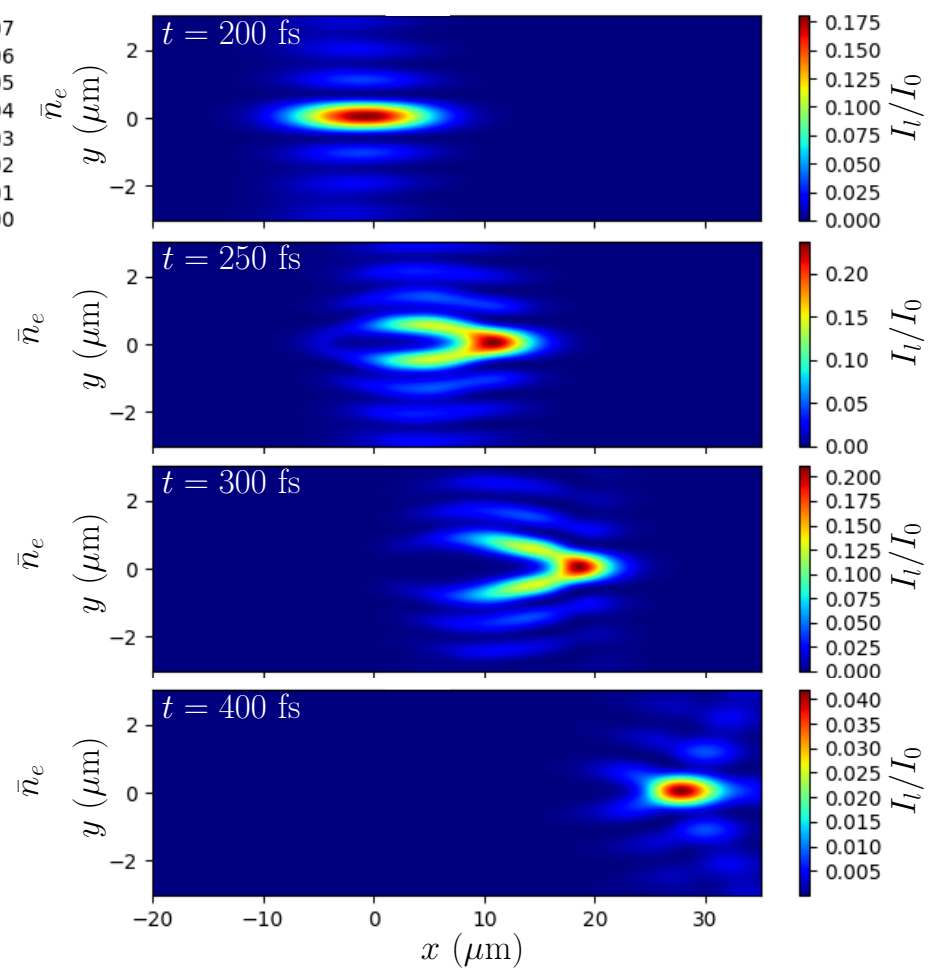

(b)

Fig. 2: Spatial and temporal evolutions in the xy plane of the free electron density (a) and laser intensity (b) in silica for $1.3 \mu \mathrm{m}$ in diameter Bessel-Gauss beam (BG3). The laser pulse comes from the left border and the laser intensity $I_{l}$ is normalized to the laser peak intensity $I_{0}=300 \mathrm{TW} / \mathrm{cm}^{2}$. The electron density $\bar{n}_{e}$ is normalized to $n_{c}=1.7 \times 10^{21} \mathrm{~cm}^{-3}$.

beam leading to stronger electron heating and ionization processes. The maximum electron density increases up to $4.3 n_{c}$. At the same time, the disturbed part of the incident beam induces a laser intensity in the edge of the absorption volume which is large enough to expand its transversal size which becomes of the order of the BG3 beam diameter.

Due to the lower $\theta$ value in the BG1 beam, the screening of the incident laser beam induced by the absorption volume formation is higher than with the BG3 beam. The laser intensity in the resulting BesselGauss beam is then smaller leading to a lower material ionization and electron heating. This is confirmed in Fig. 3 where the final calculated lattice temperature corresponding to $T_{l}=T_{e}$, is presented in the $x y$ plane for the previously considered Bessel-Gauss beams and $I_{0}=300 \mathrm{TW} / \mathrm{cm}^{2}$. The black (for BG1) and white (for BG3) curves correspond to $T_{s}=1300 \mathrm{~K}$. Material modifications or damage can be expected for temperature larger than $T_{s}$. For the BG1 beam (top pannel in Fig. 3), only a damaged material volume of $8 \mu \mathrm{m}$ in length $\left(L_{d}\right)$ where $T_{l}>T_{s}$ is obtained. In this heated matter, the averaged temperature $\bar{T}_{l}$ is $1470 \mathrm{~K}$ and the maximum temperature is $1600 \mathrm{~K}$. It is located between $x=8.8$ and $16.8 \mu \mathrm{m}$, that means in the tail of the absorption volume (see $t=400$ fs in Fig. 1(a)). The laser heating is less efficient in the first part of the absorption volume due to its tranverse size which is larger than the laser skin depth induced by the BG1 beam (of the order of $320 \mathrm{~nm}$ for $n_{e}=0.5 n_{c}$ ). For the BG3 beam (bottom pannel in Fig. 3), the temperature is greater than $T_{s}$ in a length $L_{d}$ of $30 \mu \mathrm{m}$ corresponding to the absorption volume at $t=400 \mathrm{fs}$ in Fig. 2(a). The maximum temperature is $6900 \mathrm{~K}$ and $\bar{T}_{l}=5600 \mathrm{~K}$, larger than temperatures obtained with the BG1 beam. With the same $I_{0}$ value, the final modifications of the material will be more significant with a higher $\theta$ value. This was also obtained with the BG2 beam (not shown). For $I_{0}=300 \mathbf{T W} / \mathbf{c m}^{2}$, the spatial and temporal evolutions of the electron density and laser intensity in the target for the BG2 beam is quite similar to those presented for the BG3 beam, except that the absorption volume is larger with 


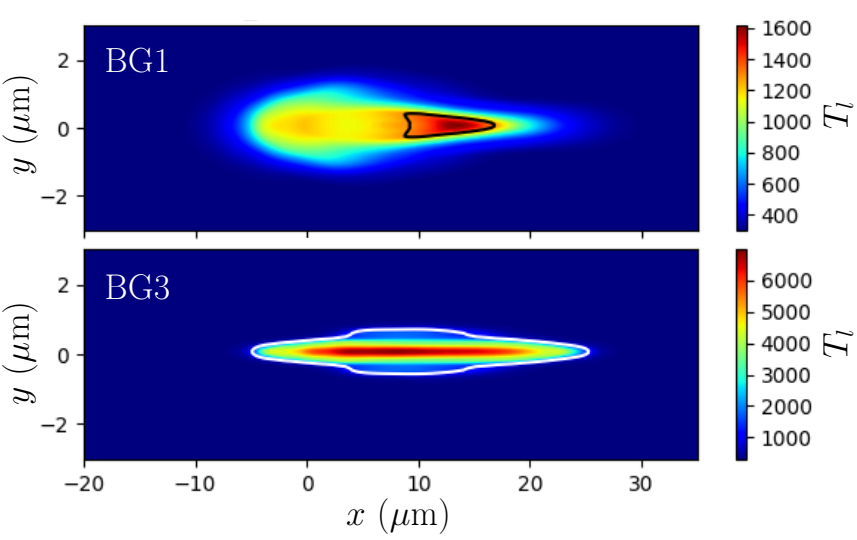

Fig. 3: Final lattice temperature in the $x y$ plane for the BG1 (top pannel) and BG3 (bottom pannel) with $I_{0}=300 \mathrm{TW} / \mathrm{cm}^{2}$. The black (for BG1) and white (for BG3) lines correspond to $T_{l}=1300 \mathrm{~K}$.
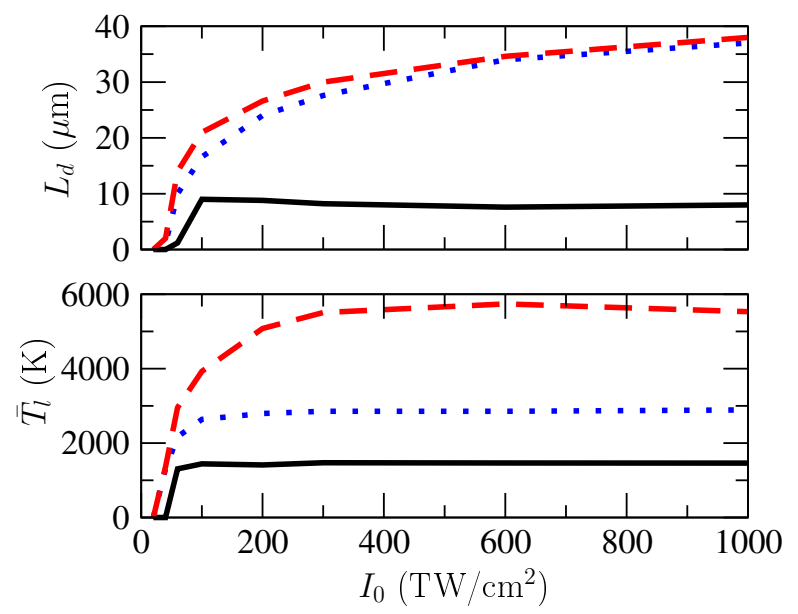

Fig. 4: Evolution of the length of the volume where $T_{l}>T_{s}$ (top pannel) and of the averaged temperature in this volume (bottom pannel) as a function of the peak laser intensity, for BG1 (black solid line), BG2 (blue dotted line) and BG3 (red dashed line) beams.

\section{the BG2 beam and therefore the final lattice temperature is lower $\left(\bar{T}_{l}=2800 \mathbf{K}\right)$.}

Figure 4 presents the calculated length $L_{d}$ of the damaged material volume where $T_{l}>T_{s}$ (top pannel) and the averaged temperature $\bar{T}_{l}$ in this volume of matter (bottom pannel), as a function of the laser peak intensity for the three Bessel-Gauss beams presented in Tab. 1. In case of BG1 beam, $\bar{T}_{l}$ becomes higher than $T_{s}$ for $60 \mathrm{TW} / \mathrm{cm}^{2}$ and $L_{d}$ starts to increase sharply with the laser intensity until $I_{0}=100 \mathrm{TW} / \mathrm{cm}^{2}$. For these laser intensities, as the maximum transverse size of the absorption volume is of the order of the skin depth, the screening effect is reduced and the volume of damaged material corresponds to the absorption vol- ume. For larger intensities, the maximum transverse size of the absorption volume increases and the damaged material volume corresponds only to the tail of the absorption volume like in Fig. 3. $L_{d}$ increases slowly while $\bar{T}_{l}$ remains constant $(\approx 1470 \mathrm{~K})$. For the two other beams, the intensity threshold is $40 \mathrm{TW} / \mathrm{cm}^{2}$. $L_{d}$ increases always with the laser intensity and tends toward a value close to $45 \mu \mathrm{m}$. However, $\bar{T}_{l}$ increases up to $2800 \mathrm{~K}$ for the BG2 beam when the laser intensity reaches $100 \mathrm{TW} / \mathrm{cm}^{2}$ and remains constant for larger values of $I_{0}$. For the BG3 beam, $\bar{T}_{l}$ increases up to $5600 \mathrm{~K}$ until $I_{0}=300 \mathrm{TW} / \mathrm{cm}^{2}$. Like with the BG1 beam, the screening effect limits the material laser heating when the laser intensity becomes higher than $100 \mathrm{TW} / \mathrm{cm}^{2}$ for the BG2 beam and $300 \mathrm{TW} / \mathrm{cm}^{2}$ for the BG3 beam. However, the undisturbed part of the incident beam is always capable to form a Bessel-Gauss beam ables to heat the matter in front of the absorption volume like in Fig 2(b).

These results show that for femtosecond Bessel-Gauss beams, the laser heating is limited to a maximum temperature even if the laser intensity increases. This maximum temperature value increases with the decrease of the Bessel-Gauss beam diameter. Such a behaviour can be expected for longer laser pulses. However, since the irradiation time is longer, the absorbed laser energy transfer toward the lattice becomes more efficient before the initiation of the impact ionization process. The electron heating is slower and the lattice energy becomes higher when the electron density becomes large enough to induce the screening effect. For these BesselGauss beams, it is easier to obtain a maximum temperature higher than the energy threshold for strong material modifications as it was experimentally observed [3].

By using larger Bessel-Gauss beam (BG1), the maximum temperature is slightly larger than the strain temperature. With such Bessel-Gauss beam, one can only expect small structure modifications like refractive index modification in the bulk of $\mathrm{SiO}_{2}$ target. The material density will be almost unchanged in the heated volume after its relaxation in the surrounding cold solid matter. Using thinner Bessel-Gauss beam allows to heat more efficiently the material along the laser pulse propagation axis due to the larger $\theta$ value. However, even if the saturated values of $\bar{T}_{l}$ are larger than the softening temperature in fused silica $(\approx 2000 \mathrm{~K})$, they are too low to induce a pressure of the order of the $\mathrm{SiO}_{2}$ bulk modulus in the heated matter (75 GPa for silica [7]) and perform voids like in tightly focused conditions with Gaussian beam [42]. For the BG2 and BG3 beams, the heated volume relaxation must be simulated to determine the resulting modifications of the material. 


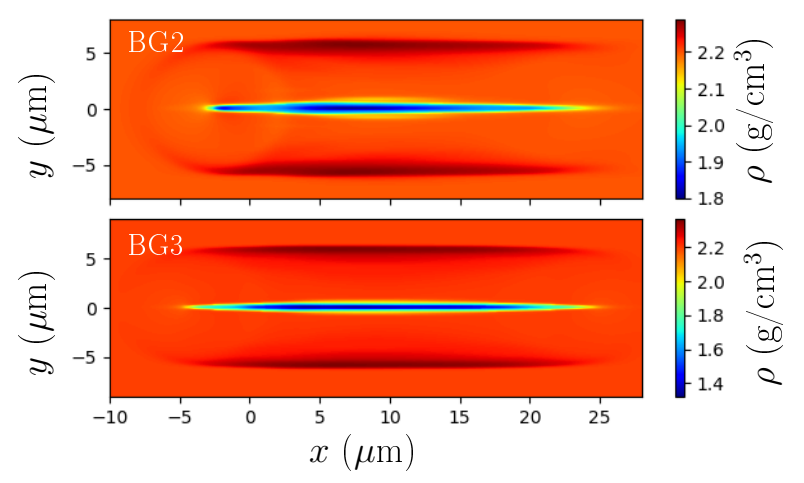

Fig. 5: 2D map of the density profile $\rho(x, y)$ at 1 ns after the BG2 and BG3 beam irradiation for $I_{0}=$ $300 \mathrm{TW} / \mathrm{cm}^{2}$.

\section{Material relaxation}

To determine the material modifications after the laser irradiation by using BG2 and BG3 beams, simulations are performed by using the $2 \mathrm{D}$ thermo-elasto-plastic model developed in Ref. [23,24] and implemented in the hydrodynamic CHIC code [21]. This model is based on the standard fluid description which has been augmented by the solid response through the elasto-plastic behavior to include the influence of the surrounding cold solid matter. Despite the simulations are 2D, correct trends are expected due to the system symetry. Deposited laser energy profiles calculated by the ARCTIC code are introduced as initial conditions in the CHIC code by assuming an isochoric heating and using the lattice temperature and pressure distributions deduced from the SESAME table 7386 for fused silica [43]. The calculated density maps $\rho(x, y)$ at $t=1 \mathrm{~ns}$ after the laser irradiation, is presented in Fig. 4 for $I_{0}=300 \mathrm{TW} / \mathrm{cm}^{2}$. In both case, a narrow filament of approximately $25 \mu \mathrm{m}$ in length and approximately $1 \mu \mathrm{m}$ in diameter where the density is lower than $2.2 \mathrm{~g} / \mathrm{cm}^{3}$ (initial solid density) and corresponding to the laser heated matter, is observed. It is surrounded by moving compressed areas where the density is higher than the initial solid density, following the sound wave launched from the heated matter. No plastic deformations resulting from the compression waves, i.e no permanent mechanical deformations of the cold solid matter, are induced in both cases. However, the matter in the filament moves toward metastable liquid states (below the liquid-vapor binodal). Thereafter, a nucleation process leading to formation of vapor bubbles (not included in our simulations) will allow it to reach a liquid-vapor equilibrium state. In the case of the BG3 beam, a uniform channel will probably be induced as the relaxation drive the matter toward a metastable state close to the critical point. In the case of BG2 beam, the final structure should be a non-uniform rarefied zone.

\section{Conclusions}

In summary, we have reported simulations of material modifications in the bulk of a dielectric by femtosecond Bessel-Gauss beams. The laser-matter interaction has been described by using a 3D Maxwell solver including the electron dynamics. The relaxation of irradiated material has been simulated by a hydrodynamic code including elasto-plastic behaviour of the solid matter. Simulations have shown that the femtosecond BesselGauss beam formation is perturbed by the laser ionized matter inducing a screening effect which leads to limit the laser energy absorption even if the incident laser energy increases. This influence is more important for the Bessel-Gauss beam with larger diameter as the $\theta$ value, the angular half aperture of the cone along which the incident gaussian beams are distributed to form the Bessel beam, is lower. For this moderate focusing conditions, the incident gaussian beam propagation is strongly disturbed by the ionized matter before to reach the interference area and form the Bessel-Gauss beam. The resulting absorbed energy in the irradiated matter is too low to induce significant modifications in the material after its relaxation. To obtain such modifications in the femtosecond regime, our results show that the Bessel-Gauss beam must be tighter focused as it was observed in experiments. The $\theta$ value becoming higher, the influence of the screening effect on the incident gaussian beam propagation is reduced. For the higher considered $\theta$ value, channel formation can be obtained. An other way to overcome the electron screening effect would be to use burst of femtosecond laser pulses with a moderate energy per pulse to limit the ionization effect, and picosecond inter-pulse time delay allowing the electron relaxation process to take place significantly before the next pulse.

Acknowledgements We acknowledge the Aquitaine Regional Council for support and funding via the MOTIF project. This work was granted access to the HPC ressources of TGCC under the allocation A0030506129 made by GENCI and the allocation 2017174175 made by PRACE.

\section{Conflict of interest}

The authors declare that they have no conflict of interest. 


\section{References}

1. M. Duocastella, C.B. Arnold, Laser Photonics Rev. 6, $607(2012)$

2. F. Courvoisier, J. Zhang, M.K. Bhuyan, M. Jacquot, J.M. Dudley, Appl. Phys. A 112, 29 (2013)

3. M.K. Bhuyan, P.K. Velpula, J.P. Colombier, T. Olivier, N. Faure, R. Stoian, Appl. Phys. Lett. 104, 021107 (2014)

4. J. Lopez, K. Mishchik, B. Chassagne, C. Javaux-Leger, C. Honninger, E. Mottay, R. Kling, in Proceedings of ICALEO pp. 60-69 (2015)

5. R.R. Gattass, E. Mazur, Nat. Photonics 2, 219 (2008)

6. E.N. Glezer, M. Milosavljevic, L. Huang, R.J. Finlay, T.H. Her, J.P. Callan, E. Mazur, Optics Letters 21, 2023 (1996)

7. E.G. Gamaly, S. Juodkazis, K. Nishimura, H. Misawa, B. Luther-Davies, L. Hallo, P. Nicolai, V.T. Tikhonchuk, Phys. Rev. B 73, 214101 (2006)

8. J. Durnin, J. J. J. Miceli, J. Eberly, Phys. Rev. Lett. 58, 1499 (1987)

9. F. Courvoisier, P.A. Lacourt, M. Jacquot, M. Bhuyan, L. Furfaro, J. Dudley, Opt. Lett. 34, 3163 (2009)

10. P.K. Velpula, M.K. Bhuyan, C. Mauclair, J.P. Colombier, R. Stoian, Opt. Engineering 53, 076108 (2014)

11. J. Artl, K. Dholakia, Opt. Comm. 177, 297 (2000)

12. V. Jarutis, R. Paskauskas, A. Stabinis, Opt. Comm. 184, $105(2000)$

13. M. Bhuyan, F. Courvoisier, P. Lacourt, M. Jacquot, R. Salut, L. Furfaro, J. Dudley, Appl. Phys. Lett. 97, $081102(2010)$

14. K. Mishchik, R. Beuton, O. Dematteo-Caulier, S. Skupin, B. Chimier, G. Duchateau, B. Chassagne, R. Kling, C. Hönninger, E. Mottay, J. Lopez, Opt. Exp. 26, 33271 (2017)

15. T.Cizmar, K. Dholakia, Opt. Exp. 17, 15558 (2009)

16. X. Liu, Q. Li, A. Sikora, M. Sentis, O. Utéza, R. Stoian, W. Zhao, G. Cheng, N. Sanner, Opt. Exp. 27, 6996 (2019)

17. L. Rapp, R. Meyer, R. Giust, L. Furfaro, M. Jacquot, P.A. Lacourt, J.M. Dudley, F. Courvoisier, Sci. Rep 6, 34286 (2016)

18. M. Bhuyan, M. Somayaji, A. Mermillod-Blondin, F. Bourquard, J. Colombier, R. Stoian, Optica 4, 951 (2017)

19. C. Arnold, S. Akturk, A. Mysyrowicz, V. Jukna, A. Couairon, T. Itina, R. Stoian, C. Xie, J. Dudley, F. Courvoisier, S. Bonanomi, O. Jedrkiewicz, P. Trapan, J. Phys. B: At. Mol. Opt. Phys. 48, 094006 (2015)

20. I. Thiele, P.G. de Alaiza Martínez, R. Nuter, A. Nguyen, L. Bergé, S. Skupin, Phys. Rev. A 96, 053814 (2017)

21. J. Breil, S. Galera, P.H. Maire, Comput. Fluids 46, 161 (2011)

22. P.H. Maire, R. Abgrall, J. Breil, R. Loubere, B. Rebourcet, J. Comput. Phys. 235, 626 (2013)

23. R. Beuton, B. Chimier, J. Breil, D. Hebert, P.H. Maire, G. Duchateau, J. Appl. Phys. 122, 203104 (2017)

24. R. Beuton, B. Chimier, J. Breil, D. Hébert, K. Mishchik, J. Lopez, P. Maire, G. Duchateau, Appl Phys A 124, 324 (2018)

25. C. Mézel, L. Hallo, A. Bourgeade, D. Hébert, V.T. Tikhonchuk, B. Chimier, B. Nkonga, G. Schurtz, G. Travaillé, Phys. Plasmas 15, 093504 (2008)

26. L.V. Keldysh, Soviet Physics JETP 20(5), $1307 \quad$ (1965). URL http://www.slac.stanford.edu/grp/arb/tn/arbvol5/AARD451.pdf

27. B.C. Stuart, M.D. Feit, S. Herman, A.M. Rubenchik, B.W. Shore, M.D. Perry, Phys. Rev. B 53, 1749 (1996)
28. J.R. Peñano, P. Sprangle, B. Hafizi, W. Manheimer, A. Zigler, Phys. Rev. E 72, 036412 (2005)

29. K. Yee, IEEE Transactions on Antennas and Propagation 14, 302 (1966)

30. J.P. Berenger, J. Comput. Phys. 114, 185 (1994)

31. J.P. Berenger, J. Comput. Phys. 127, 363 (1996)

32. F. Gori, G. Guattari, C. Padovani, Opt. Commun. 64, 491 (1987)

33. I. Thiele, S. Skupin, R. Nuter, J. Comp. Phys. 321, 1110 (2016)

34. P. González de Alaiza Martínez, G. Duchateau, B. Chimier, R. Nuter, I. Thiele, S. Skupin, V.T. Tikhonchuk, Phys. Rev. A 98, 043849 (2018)

35. X. Liu, N. Sanner, M. Sentis, R. Stoian, W. Zhao, G. Cheng, O. Utéza, Appl. Phys. A 124, 206 (2018)

36. B. Chimier, O. Utéza, N. Sanner, M. Sentis, T. Itina, P. Lassonde, F. Légaré, F. Vidal, J.C. Kieffer, Phys. Rev. B 84, 094104 (2011)

37. N. Bulgakova, R. Stoian, A. Rosenfeld, Q. Elect 40, 966 (2010)

38. N. Bulgakova, V. Zhukov, S.V. Sonina, Y. Meshcheryakov, Journal of Appl. Phys. 118, 233108 (2015)

39. A. Kaiser, B. Rethfeld, M. Vicanek, G. Simon, Phys. Rev. B 61, 11437 (2000)

40. A.Q. Wu, I.H. Chowdhury, X. Xu, Phys. Rev. B 72, $085128(2005)$

41. F. Queré, S. Guizard, P.Martin, Europhys. Lett. 56, 138 (2001)

42. S. Juodkazis, K. Nishimura, S. Tanaka, H. Misawa, E.G. Gamaly, B. Luther-Davies, L. Hallo, P. Nicolai, V.T. Tikhonchuk, Phys. Rev. Lett. 96, 166101 (2006)

43. J. Boettger, Sesame equation of state number 7386, fused quartz. Tech. rep., Los Alamos National Laboratory Report LA-11488-MS (1989) 